\title{
UNIMAXIMAL ORDERS
}

\author{
T. V. FOSSUM
}

ABSTRACT. Let $R$ be a Dedekind domain with quotient field $K$, and let $A$ be a separable $K$-algebra. An $R$-order $\Lambda$ in $A$ is said to be unimaximal if $\Lambda$ is contained in a unique maximal $R$-order in $A$. Unimaximal orders are given characterizations which are applied to determine those finite groups $G$ of order $n$ for which $R G$ is unimaximal, where $K$ is an algebraic number field containing a primitive $n$th root of unity.

1. Introduction. We assume throughout this paper that $R$ is a Dedekind domain with quotient field $K$, and that $A$ is a separable $K$-algebra. An $R$-or$\operatorname{der} \Lambda$ in $A$ is an $R$-subalgebra of $A$ which is finitely generated as an $R$-module and which contains a $K$-basis for $A$. We will follow the notation and terminology of $[5]$ concerning orders and lattices.

Definition. An $R$-order $\Lambda$ in $A$ is said to be unimaximal if $\Lambda$ is contained in a unique maximal $R$-order in $A$.

The principal tools in this paper are the characterizations of hereditary orders given by Brumer [1], Harada [2] and Jacobinski [3]. Hereditary orders correspond locally to certain subrings of rings of the type $\operatorname{End}_{D}\left(M_{D}\right)$, where $D$ is a division ring and $M_{D}$ is a finite dimensional right $D$ module; more importantly, a nonmaximal hereditary order corresponds at some prime to a subring which acts reducibly on some $M$.

Definition. Let $C$ be a unital subring of a ring $B$. We say $C$ is an irreducible subring of $B$ if for all simple left $B$-modules $M, M$ has no proper $(C, D)$-bisubmodules, where $D=\operatorname{End}_{B}(M)^{\circ}$ acts on the right of $M$.

The Jacobson radical of a ring $B$ is denoted $J(B)$. From the definition we deduce the following

Proposition. Let $C$ be an irreducible subring of $B$. If $(C+J(B)) / J(B)$ is artinian, then $J(B) \supseteq J(C)$.

Proof. Set $J=J(B)$ and $\bar{C}=(C+J) / J$. If $M$ is a simple left $B$ module, then $M$ is a nonzero left $\bar{C}$-module. Since $\bar{C}$ is artinian, $M$ contains a simple left $\bar{C}$-submodule, say $N$. Setting $D=\operatorname{End}_{B}(M)^{\circ}$, we find that $N D$ is a nonzero $(C, D)$-bisubmodule of $M$, and so by assumption $N D=M$. It follows that the annihilator of $M$ in $B$ contains the annihilator of $N$ in $C$, and therefore $J(B) \supseteq J(C)$.

Presented to the Society January 15, 1974; received by the editors October 15, 1973 and, in revised form, July 8, 1974.

AMS (MOS) subject classifications (1970). Primary 16A18, 16A26; Secondary 16A16, 16A64, 20C10, 20C20.

Key words and phrases. Maximal order, he neditary order, group algebra, Dedekind domain. 
2. Characterizations. Let $\operatorname{spec}(R)$ denote the set of prime ideals of $R$. If $P \in \operatorname{spec}(R)$ and if $X$ is a finitely generated $R$-module, we let $\hat{X}_{P}$ (or $\hat{X}$ if there is no ambiguity) denote the $P$-adic completion of $X$.

Theorem A. Let $\Lambda$ be an $R$-order in $A$, and let $\Gamma$ be a fixed maximal order containing $\Lambda$. Then the following statements are equivalent:

(a) $\Lambda$ is unimaximal.

(b) If $H$ is a hereditary $R$-order in $A$ such that $\Lambda \subseteq H$, then $H$ is maximal.

(c) If $H$ is a hereditary $R$-order in $A$ such that $\Lambda \subseteq H \subseteq \Gamma$, then $H=\Gamma$.

(d) $\hat{\Lambda}_{P}$ is an irreducible subring of $\hat{\Gamma}_{P}$ for all $P \in \operatorname{spec}(R)$.

(e) If $\Lambda^{\prime}$ is an $R$-order such that $\Lambda \subseteq \Lambda^{\prime} \subseteq \Gamma$, then $J\left(\hat{\Lambda}_{P}^{\prime}\right) \supseteq J\left(\hat{\Gamma}_{P}\right)$ for all $P \in \operatorname{spec}(R)$.

Proof. The proof will follow this scheme:

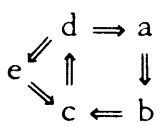

$\mathrm{a} \Rightarrow \mathrm{b}$ : Assume (a), and let $H$ be a hereditary $R$-order in $A$ such that $\Lambda \subseteq H$. By [3, Proposition 3], $H$ is the intersection of maximal orders. Since $\Lambda$ is unimaximal, $H$ must be maximal, as desired.

$\mathrm{b} \Rightarrow \mathrm{c}$ : This is obvious.

$\mathrm{c} \Rightarrow \mathrm{d}$ : Assume (d) is false, and fix some $P \in \operatorname{spec}(R)$ such that $\hat{\Lambda}$ is not irreducible in $\hat{\Gamma}$. Let $K$ be the quotient field of $R$, and set $\hat{A}=A \otimes_{K} \hat{K}$, so that $\hat{\Lambda}$ and $\hat{\Gamma}$ are $\hat{R}$-orders in $\hat{A}$. It is no loss to assume that $\hat{A}$ is simple. Since $\hat{\Gamma}$ is a maximal $\hat{R}$-order in the simple algebra $\hat{A}$ and $\hat{R}$ is complete, $\hat{\Gamma}$ has a unique simple module $M$. By hypothesis, $M$ contains a proper $(\hat{\Lambda}, D)$ submodule, say $N$, where $D$ is the endomorphism ring of $M$ over $\hat{\Gamma}$. By [3, Proposition 2], $\hat{H}=\{x \in \hat{\Gamma}: x N \subseteq N\}$ is a nonmaximal hereditary order in $\hat{\Gamma}$, and clearly $\hat{\Lambda} \subseteq \hat{H}$. It is now easy to construct a nonmaximal hereditary order $H$ such that $\Lambda \subseteq H \subseteq \Gamma$. Thus (c) is false, as desired.

$\mathrm{d} \Rightarrow \mathrm{a}$ : Let (d) hold. It is enough to assume that $A$ is simple and $R$ is complete. It follows that $J=J(\Gamma)$ is the unique maximal two-sided ideal of $\Gamma$. Let $\Gamma^{\prime}$ be a maximal order containing $\Lambda$, and set $\Lambda^{\prime}=\Gamma \cap \Gamma^{\prime}$. Clearly $\Lambda^{\prime} \supseteq \Lambda$. Since $R$ is complete, (d) implies that $\Lambda$, and hence $\Lambda^{\prime}$, is irreducible as a subring of $\Gamma$, so it follows that $\left(\Lambda^{\prime}+J\right) / J$ is simple. Since $J$ is an invertible $\Gamma$-ideal, there exists an integer $n$ such that $J^{n} \Gamma^{\prime} \subseteq \Gamma$ but $J^{n} \Gamma^{\prime} \nsubseteq J J$, and we set $I=J^{n} \Gamma^{\prime}$. Notice that $I$ is a left ideal of $\Gamma$. Since $\Gamma^{\prime}$ is a maximal order, the right order $O_{r}(I)$ of $I$ is $\Gamma^{\prime}$. But then $\Gamma \cap O_{r}(I)=$ $\Gamma \cap \Gamma^{\prime}=\Lambda^{\prime}$, and therefore $I$ is a two-sided ideal in $\Lambda^{\prime}$. It follows that $(I+J) / J$ is a two-sided ideal in $\left(\Lambda^{\prime}+J\right) / J$, and since the latter is a simple ring, either $I+J=J$ or $I+J=\Lambda^{\prime}+J$. The first possibility is ruled out 
since $I \not \subset J$, and hence

$$
I+J=\Lambda^{\prime}+J
$$

Now $I$ is a left ideal of $\Lambda^{\prime}+J$, and $J$ is contained in the radical of $\Lambda^{\prime}+J$, so Nakayama's lemma applied to $(*)$ shows that $I=\Lambda^{\prime}+J$. In particular, $1 \in I$. Inasmuch as $I$ is a left ideal of $\Gamma$, we find that $I=\Gamma$, whence $\Gamma=$ $O_{r}(I)=\Gamma^{\prime}$, showing that (d) implies (a).

$\mathrm{d} \Rightarrow \mathrm{e}$ : This follows directly from the Proposition in $\S 1$.

$\mathrm{e} \Rightarrow \mathrm{c}$ : Let (e) hold, and assume $H$ is a hereditary $R$-order such that $\Lambda \subseteq H \subseteq \Gamma$. By assumption, $J\left(\hat{H}_{P}\right) \subseteq J\left(\hat{\Gamma}_{P}\right)$ for all $P \in \operatorname{spec}(R)$. Jacobinski's characterization [3, Theorem 1] of hereditary orders implies that $H=\Gamma$, as desired.

This concludes the proof of the theorem.

It is interesting to single out the following special case:

Corollary. Let $\Gamma$ and $\Gamma^{\prime}$ be distinct maximal R-orders in $A$. Then $\Gamma \cap$ $\Gamma^{\prime}$ is contained in a nonmaximal hereditary order.

Example. Let $K$ be the field of rational numbers, and let $\mathbf{Z}_{(2)}$ be the localization of the ring $\mathrm{Z}$ of integers at the prime 2. The polynomial $x^{2}+x$ +1 is irreducible over the residue class field $\mathbf{Z} / 2 \mathbf{Z}$ of $\hat{Z}_{(2)}$, and therefore if $\Lambda$ is any $Z_{(2)}$-order in $K_{2}$ (the ring of two-by-two matrices over $K$ ) which contains the companion matrix $\left(\begin{array}{cc}0 & 1 \\ -1 & -1\end{array}\right)$ of $x^{2}+x+1$, then $\Lambda$ is unimaximal.

3. Group algebras. We use a theorem in modular character theory together with the result of the previous section to characterize those group algebras (with suitable restrictions on the ground ring) which are unimaximal.

A finite group $G$ is said to be $p$-nilpotent ( $p$ a rational prime) if $G$ contains a normal subgroup $N$ of order relatively prime to $p$ such that $G / N$ is a $p$-group.

Theorem B. Let $G$ be a finite group of order $n$, let $K$ be an algebraic number field containing a primitive nth root of unity, and let $R$ be a Dedekind domain with quotient field $K$. Then $R G$ is unimaximal in $K G$ if and only if

(1) $G$ is p-nilpotent, and

(2) the Sylow p-subgroups of $G$ are abelian for all rational pirmes $p$ which are nonunits in $R$.

Proof. Let $\Gamma$ be a maximal $R$-order in $K G$ which contains $\Lambda=R G$, and fix $P \in \operatorname{spec}(R)$. Let $p$ be the rational prime in $P$. Since $K$ is a splitting field for $K G$, it follows that $J(\hat{\Gamma})=\hat{P} \hat{\Gamma}$. Now let $M$ be an irreducible (in the sense of lattices) left $\hat{\Gamma}$-lattice, so that $M$ is also an irreducible left $\hat{\Lambda}$-lattice (see [5, Chapter IV, Lemma 1.13]). One easily checks that $M / \hat{P} M$ is a simple left $\hat{\Gamma}$-module, and every simple left $\hat{\Gamma}$-module can be obtained in this way. Clearly $\operatorname{End}_{\hat{\Gamma}}(M / \hat{P} M)=\hat{R} / \hat{P}$, so any $(\hat{\Lambda}, \hat{R} / \hat{P})$-bisubmodule of $M / \hat{P} M$ is 
simply a left $\hat{\Lambda}$-submodule. From Theorem $A$, we see that $\hat{\Lambda}$ is unimaximal if and only if each such $M / \hat{P} M$ is a simple $\hat{\Lambda}$-module. Noting that $\hat{\Lambda} / \hat{P} \hat{\Lambda}=$ $F G$, where $F=\hat{R} / \hat{P}$, we can apply a theorem of Richen [4] which says that $M / \hat{P} M$ is a simple $\hat{\Lambda}$-module for all such $M$ if and only if $G$ is $p$-nilpotent and the Sylow $p$-subgroups are abelian. This concludes the proof.

Corollary. Let $G$ be a finite group of order $n$, let $K$ be an algebraic number field containing a primitive nth root of unity, and let $R$ be the ring of algebraic integers in $K$. Then $R G$ is unimaximal in $K G$ if and only if $G$ is abelian.

Proof. Note first that every rational prime $p$ is a nonunit in $R$. From Theorem $\mathrm{B}$ we see that $R G$ is unimaximal in $K G$ if and only if $G$ is $p$-nilpotent with abelian Sylow $p$-subgroups for all primes $p$. One can readily show that this is equivalent to $G$ being abelian.

Example. Let $G=S_{3}$, the (nonabelian) symmetric group on 3 letters, of order 6 . Let $K$ be the field of rational numbers, and let $R=\mathrm{Z}_{(3)}$. Since $G$ is not 3-nilpotent and 3 is not a unit in $R, R G$ is not unimaximal. We give an explicit proper hereditary order containing $R G$. Write $K G=K \oplus K \oplus K_{2}$, a direct sum of full matrix algebras over $K$. Now $G$ is generated by the two 2cycles (12) and (13). The correspondence

$$
(12) \rightarrow\left(1,-1,\left(\begin{array}{rr}
1 & 0 \\
1 & -1
\end{array}\right)\right), \quad(13) \rightarrow\left(1,-1,\left(\begin{array}{rr}
1 & 3 \\
0 & -1
\end{array}\right)\right)
$$

defines an embedding of $R G$ into $K G$. Now the elements of $K G$ of the form $\left(a, b,\left(\begin{array}{cc}c & 3 d \\ e & f\end{array}\right)\right.$, where $a, b, c, d, e, f \in R$, is a proper hereditary order in $K G$ which clearly contains $R G$. From this the reader may determine two distinct maximal $R$-orders in $K G$ which contain $R G$.

\section{REFERENCES}

1. A. Brumer, Structure of herediatry orders, Bull. Amer. Math. Soc. 69 (1963), $721-724$. MR $27 \# 2543$.

2. M. Harada, Hereditary orders, Trans. Amer. Math. Soc. 107 (1963), 273-290. MR $27 \# 1474$.

3. H. Jacobinski, Two remarks about hereditary orders, Proc. Amer. Math. Soc. 28 (1971), 1-8. MR $42 \# 7688$.

4. F. Richen, Decomposition numbers of p-solvable groups, Proc. Amer. Math. Soc. 25 (1970), 100-104. MR $40 \# 7356$.

5. K. W. Roggenkamp and V. Huber-Dyson, Lattices over orders. I, Lecture Notes in Math., vol. 115, Springer-Verlag, Berlin and New York, 1970. MR 44 \#247a.

DEPARTMENT OF MATHEMATICS, UNIVERSITY OF UTAH, SALT LAKE CITY, UTAH 84112

DIVISION OF SCIENCE, UNIVERSITY OF WISCONSIN-PARKSIDE, KENOSHA, WISCONSIN 53140 